\title{
On centrally coordinated voltage support in a self-dispatch system
}

\section{Conference Paper}

Author(s):

Zigkiri, Alexandra; Scherer, Marc; Gallmann, Jannick; Roduner, Cattia; Cuderman, Luka

Publication date:

2017-07

Permanent link:

https://doi.org/10.3929/ethz-b-000412458

Rights / license:

In Copyright - Non-Commercial Use Permitted

Originally published in:

https://doi.org/10.1109/PESGM.2017.8273910 


\title{
On Centrally Coordinated Voltage Support in a Self-Dispatch System
}

\author{
Alexandra Zigkiri, Marc Scherer, Jannick Gallmann, Cattia Roduner, Luka Cuderman \\ Swissgrid Ltd., Switzerland \\ \{ alexandra.zigkiri, marc.scherer, jannick.gallmann, cattia.roduner, luka.cuderman $\} @$ swissgrid.ch
}

\begin{abstract}
Advanced optimization techniques for voltage support planning in self-dispatch systems present several opportunities for transmission system operators. Before an initial implementation of such techniques in daily operation, the expected benefits are usually assessed. However, such an assessment is subject to high uncertainties related to the expected participants' behavior. A proper assessment of consequences of applying optimization for voltage support planning requires an ex-post analysis with several years of operational data. This paper contains a breakdown of relevant impacts based on five years of operational data from the Swiss transmission system. It discusses the challenges of increasing penetration of renewable energy sources on the transmission system voltages and identifies drawbacks of the voltage support planning applied in Switzerland. Finally, it quantifies potential improvements, such as moving the voltage planning closer to real-time in order to account for intraday market activity.
\end{abstract}

Index Terms - Voltage support, voltage optimization, RES integration.

\section{INTRODUCTION}

The successful operation of interconnected electric power systems requires not only the global matching of generation and consumption but also local provision of reactive power to ensure voltage stability. Together with frequency control, voltage support represents an important part of ancillary services and is crucial for security of supply [1]. In the transmission system, it is considered an ancillary service; however, due to local characteristics of voltage, reactive power resources are generally not subject to a market-driven procurement in Europe. Instead, voltage support is usually organized bilaterally between system operators and providers [2]-[4].

In highly meshed power systems such as the Continental European system the main challenge of voltage support is the coordination of reactive power resources. A high degree of meshing inherently mitigates potential dynamic voltage stability issues, and the design of voltage support lies in the responsibility of national Transmission System Operators (TSOs). Set-points for voltages or reactive power are usually provided to reactive power resources such as power plants or Flexible AC Transmission Systems (FACTS) connected to the transmission grid. The objective functions for defining setpoints and instructions vary across the European power system depending on available infrastructure; however, a common target is the minimization of active power losses in the transmission system [5]-[8]. Additionally, a coordinated voltage support concept aims to minimize the reactive power exchange with neighboring TSOs and distribution systems [9].

This paper focuses on voltage support in the high voltage transmission system. On the example of Switzerland, the potential and challenges of centrally coordinated voltage support are elaborated and analyzed. Switzerland features a deregulated electricity market, which is a typical setup for Europe [10]. The Swiss TSO is responsible for the organization of reactive power management in the transmission system and its connection points [11]. Switzerland developed a concept for coordinated voltage support of the $220 / 380-\mathrm{kV}$ transmission system that enables the participation of both, power plants connected to the transmission system as well as connected distribution systems [12].

This paper is organized as follows: Section II elaborates on voltage basics for analyzing the Swiss concept. Section III presents the analyzed data. Section IV assesses the performance of centrally coordinated voltage support. Section V presents the results, discusses the implications of optimization uncertainties on system operation and identifies enhancements. Finally, Section VI is devoted to conclusions and perspectives.

\section{Characteristics of Voltage Support}

In a transmission system, four parties are involved in voltage support. The first party is the transmission system itself, as TSOs do not own production facilities and therefore require coordinated reactive power management. The second party are the power plants which are directly connected to the transmission system and are able to control their reactive power production. Third, distribution grids and end-consumers directly connected to the transmission system influence the voltage with their reactive power exchange; however, their controllability is limited. Finally, neighboring transmission systems can significantly influence the voltage by inducing unexpected transit flows and inadvertent reactive power flows on the tie-lines.

\section{A. Swiss Voltage Support Concept}

In the Swiss concept, the transmission nodes can be classified as either actively or passively controlled. Actively controlled transmission nodes contribute to voltage control by tracking a set-point, $U_{\text {set }}$, which they receive from the TSO, by adjusting their actual voltage, $U_{\text {act }}$, through absorption or production of reactive power. They are incentivized to follow these set-points by receiving financial compensation. Actively controlled transmission nodes mainly consist of nodes with direct power plant connections. Due to the fact that power plants are the main resource of reactive power, all power plants directly connected to the transmission system are required to actively support the voltage.

Distribution grids and end-consumers can be active or passive participants [12]. In active mode, they are expected to support the voltage in the same way as power plants. Using the 


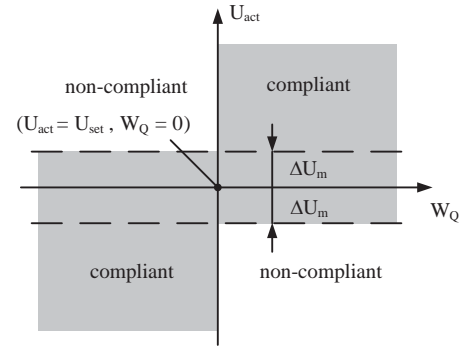

(a) Active transmission nodes (including an insensitivity $U_{\mathrm{m}}$ ).

Fig. 1: Compliance visualization for active and passive participation.

flexibility of distributed generation, for example, run-of-river power plants or transformer taps, a predefined voltage set-point can be supported by deliberately exchanging reactive power at the respective connection point to the transmission system. In passive mode, the parties are requested to limit their reactive power exchange, $W_{\mathrm{Q}}$, relative to their active power exchange, $W_{\mathrm{P}}$, at the connection point. The incentive for such a behavior is given by a default penalty rate, which applies if the reactive power exchange is beyond a Power Factor (PF) of 0.9 or a free exchange band, $W_{\mathrm{Q}, \mathrm{lim}}$. Figure 1 summarizes the compliance requirements for active and passive participation.

Since 2009, Swissgrid has used a Day-Ahead Reactive Planning (DARP) process in order to provide day-ahead voltage schedules to actively controlled transmission nodes. The dayahead voltage schedules contain the individual hourly set-point values for all active transmission system nodes. In addition, DARP provides the expected day-ahead voltage profile of the passive transmission system nodes. The main input data for DARP consists of the hourly Day-Ahead Congestion Forecast (DACF) models which contain the Continental European power flow forecast for the following day. DARP performs an Optimal Power Flow (OPF) for all 24 hours in a consecutive manner, seeking to optimize the costs for active power losses in the Swiss transmission system as well as the costs for financial compensation of the deployed reactive power by the Swiss active participants for voltage control purposes. It also considers an upper and a lower voltage limit for every connection point and an upper and a lower reactive power exchange limit for every neighboring control area.

\section{B. Experience from Real System Operation}

The increasing penetration of Renewable Energy Sources (RES) has intensified voltage support challenges in the Swiss transmission system. Figure $2 \mathrm{a}$ shows the significant increase in Photovoltaic (PV) production in Switzerland since 2011. Due to the increased PV production and the subsequently high distribution grid voltages, passive transmission nodes inject more reactive power into the grid. Figure $2 b$ presents the cumulative injection of reactive power by the passive transmission nodes. We observe that since 2011 the absorption of reactive power by passive nodes has decreased and starting from 2013 passive nodes inject reactive power into the grid. Furthermore, the increased PV production also results in a lower consumption of active

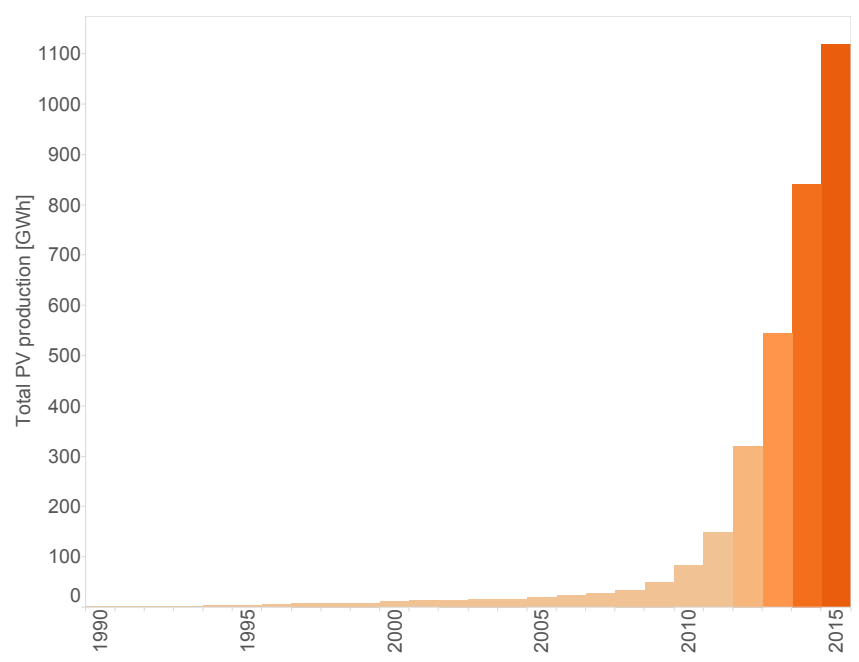

(a) Evolution of PV production in Switzerland.

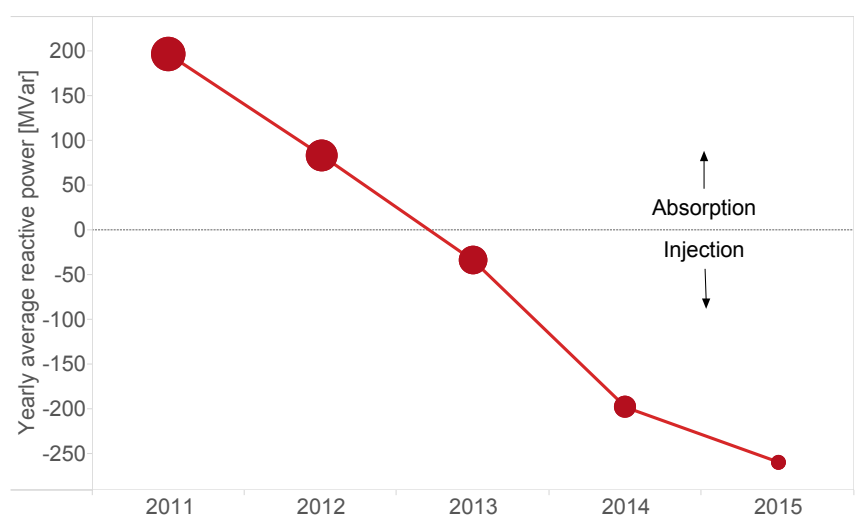

(b) Reactive power production of passive nodes.

Fig. 2: Increasing PV production affects the reactive power exchange of passive participants.

power from the transmission grid, leading to a lower vertical load in the transmission system and less line loading.

The increased injection of reactive power by the passive nodes together with the lower system load and the fact that overhead Swiss transmission lines typically operate below the surge impedance loading, results in high voltages in the Swiss transmission system. In particular, during off-peak hours and summer holidays, overvoltages occur in different parts of the grid. Note that a voltage violation, either overvoltage or undervoltage, is classified according to the severity as yellow, orange, or red. A yellow violation does not trigger any remedial action, whereas for orange and red violations, operators are required to activate countermeasures, such as additional reactive power provision from power plants in the concerned area. Figure 3 presents the areas of the system with frequent overvoltages; the majority of these violations occurs in the $220 \mathrm{kV}$ grid. In these areas the total amount of voltage violations has increased since 2011. At the same time, we observe very few actively controlled transmission nodes in the concerned areas.

On the other hand, in the areas where passive nodes 


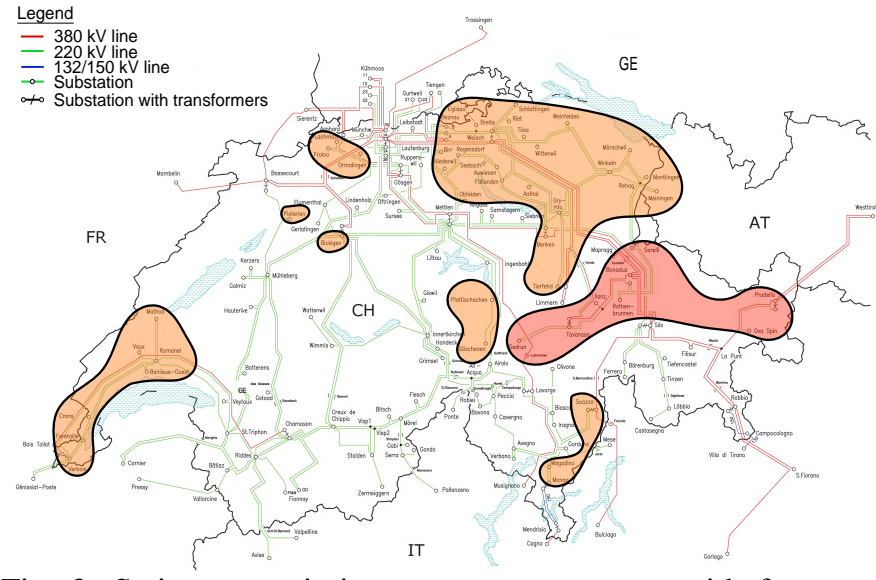

Fig. 3: Swiss transmission system map: areas with frequent overvoltages.

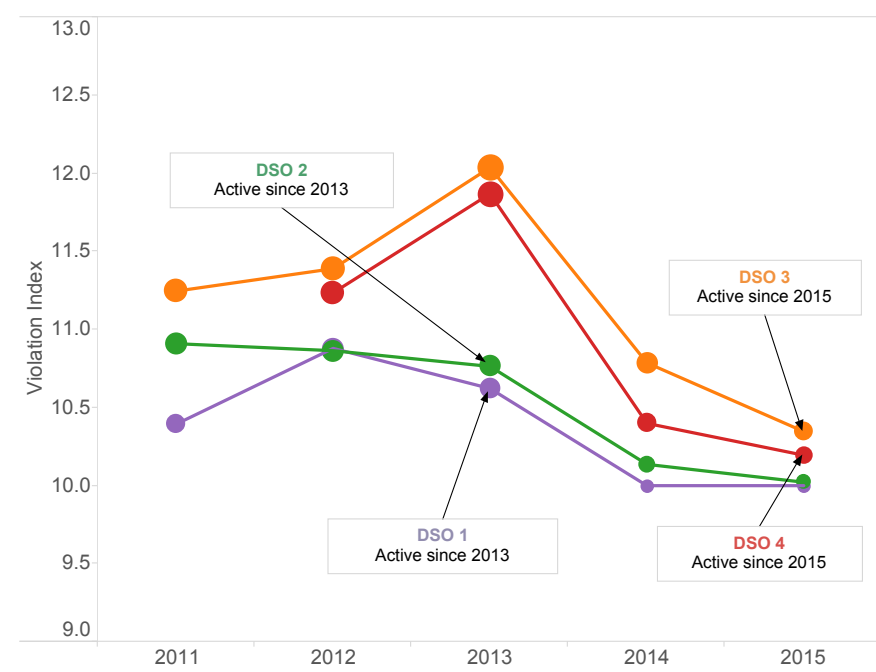

Fig. 4: Violation index for DSOs that switched from passive to active participation.

have switched from passive to active participation, voltage violations have decreased. To quantify this effect, we use the following violation index:

$$
100 \cdot \frac{0.6 N_{\mathrm{r}}+0.3 N_{\mathrm{o}}+0.1 N_{\mathrm{y}}}{N_{\mathrm{r}}+N_{\mathrm{o}}+N_{\mathrm{y}}},
$$

where $N_{\mathrm{r}}, N_{\mathrm{o}}$ and $N_{\mathrm{y}}$ are the number of red, orange and yellow voltage violations on a 15-minute basis, respectively. Figure 4 presents this violation index for four $220 \mathrm{kV}$ nodes with overvoltages, where the connecting Distribution System Operators (DSOs) feature an active participation. We observe that starting from the year a DSO has turned active, the violation index decreases.

\section{INVESTIGATION SETUP}

Since the voltage control concept of the Swiss transmission system is mainly based on DARP, it is important to ensure that the results of DARP lead to an efficient and reliable system operation. In the following, we investigate the differences between DARP results and the actual behavior of the Swiss

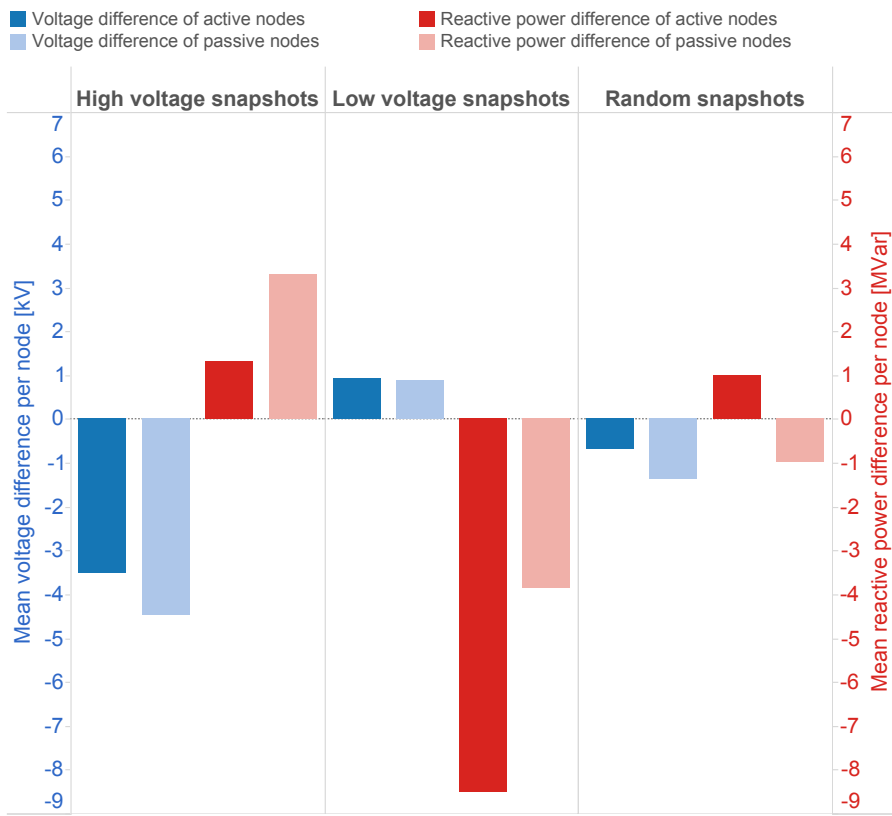

Fig. 5: DARP vs. real-time: average differences across nodes and snapshots.

transmission system along two dimensions: (i) voltages and (ii) reactive power. We use historical data for the period 2014 to 2016 corresponding to three different situations in the Swiss transmission system: (a) High voltages, (b) Low voltages, and (c) Random data, i.e. snapshots, where voltages of all transmission system nodes were generally kept within the normal operating range. We analyze fourteen high voltage snapshots, five low voltage snapshots and ten random snapshots. High voltages are the focus of the subsequent analysis, due to the high frequency of their occurrence in the Swiss transmission system, as described in Section II-B.

\section{Performance Assessment}

In this Section, we compare the results of DARP against the actual voltage behavior of the Swiss transmission system. We also investigate the use of an Intra-Day Reactive Planning (IDRP), where the voltage optimization takes place one hour before real-time. We compare the results of DARP against the results of IDRP, in terms of differences between forecast and real-time voltages. All simulations were performed in SCOPE, the optimization engine used by the DARP process.

\section{A. Planned Optimization vs. Actual Voltage Behavior}

We consider the differences of the real-time voltages and reactive power injection or absorption from the respective DARP results for each node: $V_{\mathrm{DARP}}-V_{\mathrm{RT}}$ and $Q_{\mathrm{DARP}}-Q_{\mathrm{RT}}$. Note that from a transmission grid perspective positive reactive power implies reactive power absorption, whereas negative reactive power implies reactive power injection. Thus, when the reactive power difference, $Q_{\mathrm{DARP}}-Q_{\mathrm{RT}}$, is positive, DARP forecasts that nodes absorb more (inject less) reactive power than the real-time values, whereas when this difference is negative, DARP forecasts a lower absorption (higher injection) of reactive power, compared to real-time values. 


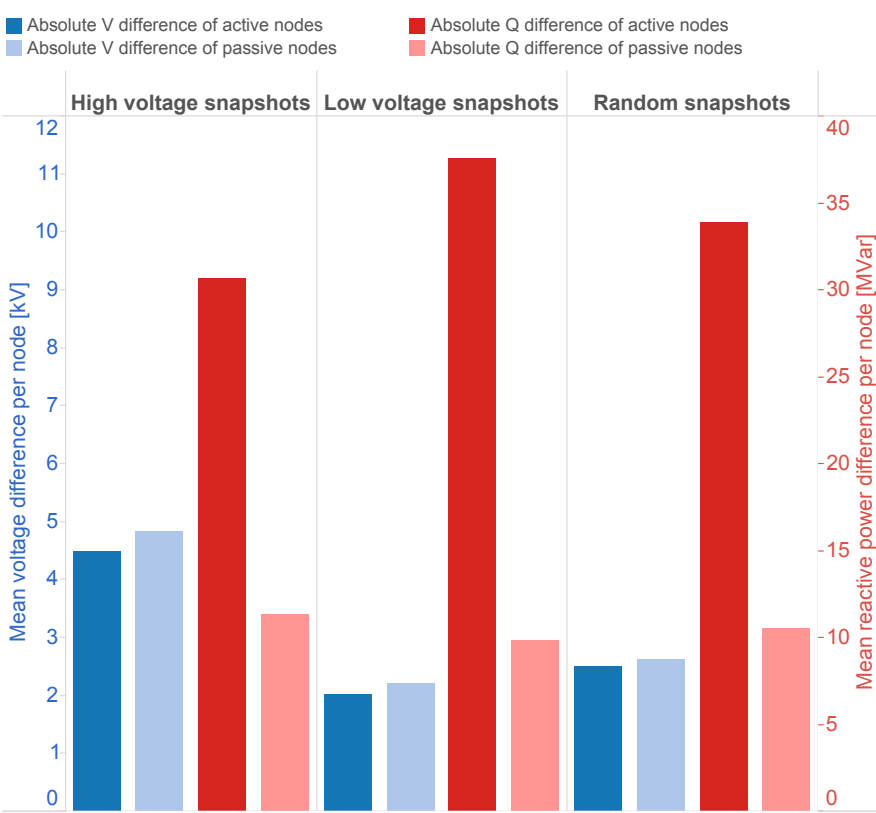

Fig. 6: DARP vs. real-time: average absolute differences across nodes and snapshots.

Figure 5 presents the average of these differences across all passive and active nodes, and all snapshots. For all high voltage snapshots, voltages forecast by DARP are lower than real-time voltages. Simultaneously we observe a higher reactive power absorption (lower injection) of passive nodes forecast by DARP compared to real-time. The same holds for active nodes, however in this case the differences are much lower. On the other hand, regarding the low voltage snapshots, voltages forecast by DARP are higher and both active and passive nodes absorb less (inject more) reactive power than in real-time. Hence, in real-time both active and passive nodes absorb more (inject less) reactive power, herewith contributing to the lower voltages. Finally, for random snapshots, voltages forecast by DARP are lower than in real-time, due to the fact that high voltages generally occur more often in the Swiss transmission system. The reactive power differences are not systematically positive or negative, neither for active nor for passive nodes, resulting in low netted deviations.

Figure 6 depicts the average of the absolute differences across all passive and active nodes, and all snapshots. The largest voltage difference amounts to $4.8 \mathrm{kV}$ and corresponds to the high voltage snapshots and passive nodes, whereas the largest reactive power difference amounts to $11.2 \mathrm{MVar}$ and corresponds to the low voltage snapshots and active nodes.

\section{B. Day-Ahead vs. Intraday Optimization}

In the following, we consider the use of an Intraday Reactive Planning (IDRP) with identical functionality as DARP. The difference lies in the input data. Again, we consider the voltage and reactive power differences, $V_{\mathrm{DARP}}-V_{\mathrm{RT}}$ and $Q_{\mathrm{DARP}}-Q_{\mathrm{RT}}$, across all studied snapshots. Table I presents the results. We observe that IDRP achieves results that are closer to the realtime values than DARP.

\begin{tabular}{lll}
\hline & DARP & IDRP \\
\hline Voltage difference $[\mathrm{kV}]$ & 1.17 & 1.06 \\
\hline Reactive power difference $[\mathrm{MVar}]$ & 5.45 & 5.06 \\
\hline
\end{tabular}

TABLE I: Comparison between DARP and IDRP.

Figures 7 and 8 present the respective average and absolute voltage and reactive power differences across all nodes and snapshots. The largest absolute voltage difference amounts to $4 \mathrm{kV}$ and corresponds to the high voltage snapshots and active nodes, whereas the largest absolute reactive power difference amounts to $10.8 \mathrm{MVar}$ and corresponds to the random snapshots and active nodes.

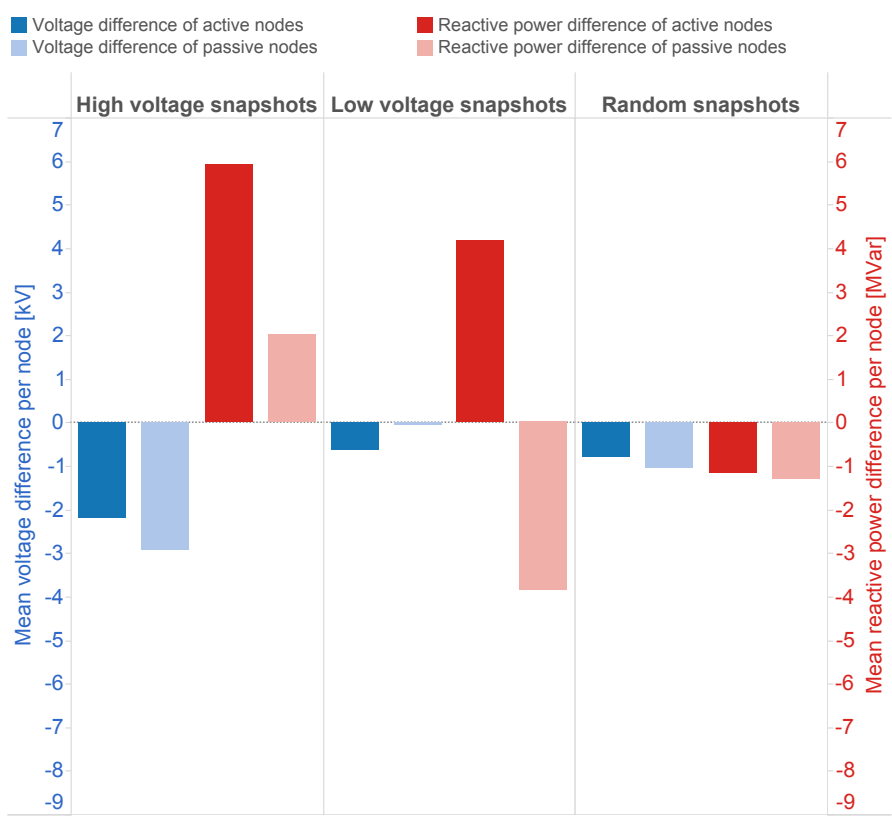

Fig. 7: IDRP vs. real-time: average differences across nodes and snapshots.

We observe that the differences between DARP and IDRP are pronounced for high voltage snapshots. Note that for these snapshots, active nodes are not able to follow the DARP setpoints; hence, the active nodes cannot absorb the forecast amounts of reactive power in order to bring the voltages to the requested set-points. The fact that IDRP values are closer to the real-time values shows that the IDRP set-points are higher, while maintaining the voltages at acceptable levels. On the other hand, regarding low and random voltage snapshots, the differences are small. The main identified causes are the small sample size and other influencing parameters, like the grid loading and the exchanges with neighboring countries. Another important factor is that, in reality, active nodes have not received the IDRP set-points, and are thus incentivized to follow set-points originally calculated by DARP.

\section{DISCUSSION}

Voltage violations occur frequently in the Swiss transmission system. Compulsory voltage support participation for transmission-connected power plants as well as increased incentives for active participation of distribution grids have 


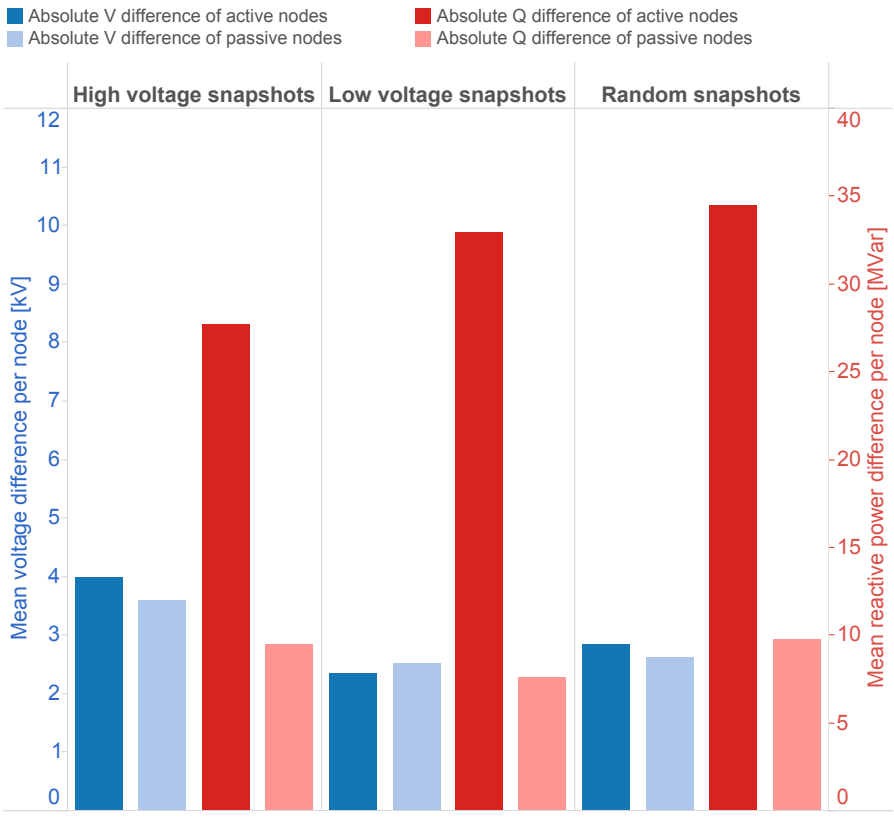

Fig. 8: IDRP vs. real-time: average absolute differences across nodes and snapshots.

increased the amount of reactive power procured by the Swiss TSO, which should in turn reduce the total amount of voltage violations. In contrast, we observe rising amounts of overvoltages. This can be attributed to the increased PV penetration in distribution grids that leads to higher distribution grid voltages. Another factor is the changed loading of the system, mainly influenced by the lower active power consumption of distribution grids and by different energy market trading patterns. DARP should consider these changes and present viable voltage set-points for active participants; however, the results of DARP are highly dependent and limited by the quality of the input data. Increasing traded volumes in the intraday markets as well as modeling of passive nodes can thus result in high deviations between the forecast day-ahead and real-time system behavior. By moving to an intraday reactive planning process, we can reduce the respective deviations and obtain better results, especially for high voltage situations, for which a systematic offset has been outlined in Section IV-A. Using IDRP can slightly decrease the voltage and reactive power differences between the planning stage and real-time values across all analyzed snapshots and nodes of the Swiss transmission system. In the areas of the Swiss transmission system featuring few active participants, an investigation of other options is required: either the introduction of higher financial incentives for passive nodes to become active or the installation of compensation devices.

\section{Closing Remarks}

\section{A. Conclusion}

This paper demonstrates, based on analysis of five years of operational data, the benefits and challenges of using optimization for voltage planning in a transmission system and suggests improvements for the identified shortcomings of the current setup. We elaborate on the experience from system operation and show that increased PV production in distribution grids results in higher voltages in the transmission system. We show that the regions of the Swiss transmission system with frequent voltage violations feature mainly passively controlled nodes, whereas the overvoltages have decreased in the regions where previous passive nodes switched to actively controlled nodes. We conclude that the Swiss voltage support concept works, but is limited through the reactive power provision capabilities of DSOs in critical regions. Moreover, moving the reactive planning process closer to real-time operation and herewith acknowledging the intraday market improves the voltage setpoint calculation, in particular for the common case of high real-time voltages.

\section{B. Outlook}

For a large-scale implementation of a flexible concept, similar to to the Swiss one, the incentives of DSOs for switching from passive to active participation need further investigation. In particular, the geographical location should be considered during pre-qualification in order to ensure distributed voltage support throughout the entire transmission system. The coordination with neighboring TSOs should also be further improved. Finally, the installation of FACTS owned by the TSO is an alternative to acquiring flexibility from distribution systems; however, the costs of this option need to be assessed against the costs and capabilities of potential active DSO participants.

\section{REFERENCES}

[1] P. S. Kundur, N. J. Balu, and M. G. Lauby, Power System Stability and Control. McGraw-Hill, Inc., 1994.

[2] B. Kirby and E. Hirst, "Unbundling Electricity: Ancillary Services," Power Engineering Review, IEEE, vol. 16, no. 6, pp. 5-6, June 1996.

[3] Y. G. Rebours, D. S. Kirschen, M. Trotignon, and S. Rossignol, "A Survey of Frequency and Voltage Control Ancillary Services - Part I: Technical Features," IEEE Transactions on Power Systems, vol. 22, no. 1 , pp. 350-357, February 2007.

[4] _ - "A Survey of Frequency and Voltage Control Ancillary Services - Part II: Economic Features," IEEE Transactions on Power Systems, vol. 22, no. 1, pp. 358-366, February 2007.

[5] E. L. Miguélez, F. M. E. Cerezo, and L. R. Rodríguez, "On the assignment of voltage control ancillary service of generators in Spain," IEEE Transactions on Power Systems, vol. 22, no. 1, pp. 367-375, February 2007.

[6] A. D. Papalexopoulos and G. A. Angelidis, "Reactive power management and pricing in the California market," in IEEE Mediterranean Electrotechnical Conference (MELECON 2006), May 2006, pp. 902 905.

[7] T. Margotin, P. Juston, M. P. Houry, G. Morales, P. Bertolini, and D. Burnel, "Reactive power support on the French power system: generation units performance monitoring," in IEEE Mediterranean Electrotechnical Conference (MELECON 2006), May 2006, pp. 906-909.

[8] S. Corsi, P. Marannino, N. Losignore, G. Moreschini, and G. Piccini, "Coordination between the reactive power scheduling function and the hierarchical voltage control of the ehv enel system," IEEE Transactions on Power Systems, vol. 10, no. 2, pp. 686-694, May 1995.

[9] OpHB-Team, "Policy 4: Co-ordinated Operational Planning," in UCTE Operation Handbook. ENTSO-E, Version 2.7, December 2014.

[10] M. Scherer, O. Haubensak, and T. Staake, "Assessing distorted trading incentives of balance responsible parties based on the example of the Swiss power system," Energy Policy, vol. 86, pp. 792-801, 2015.

[11] M. Geidl, "Implementation of coordinated voltage control for the Swiss transmission system," in 15th IEEE Mediterranean Electrotechnical Conference (Melecon 2010), April 2010, pp. 230-236.

[12] M. Zerva and M. Geidl, "Contribution of active distribution grids to the coordinated voltage control of the Swiss transmission system," in Power Systems Computation Conference (PSCC), August 2014, pp. 1-8. 